\title{
Physics of Clocks in Absolute Space-Time
}

\author{
Edwin Eugene Klingman (1) \\ Cybernetic Micro Systems, Inc., San Gregorio, CA, USA \\ Email:klingman@geneman.com
}

How to cite this paper: Klingman, E.E. (2020) Physics of Clocks in Absolute SpaceTime. Journal of Modern Physics, 11, 19501968.

https://doi.org/10.4236/jmp.2020.1112123

Received: November 6, 2020

Accepted: December 13, 2020

Published: December 16, 2020

Copyright (C) 2020 by author(s) and Scientific Research Publishing Inc. This work is licensed under the Creative Commons Attribution International License (CC BY 4.0).

http://creativecommons.org/licenses/by/4.0/ (c) (i) Open Access

\begin{abstract}
20th century physics experimentally established beyond doubt the fact that moving clocks read differently from "static" clocks. This fact is typically interpreted as support for special relativity. On the other hand, the same century produced proof that clocks at various locations in the gravitational field also read differently, and this fact is explained by general relativity, which is, in general, not Lorentz transformable. This paper establishes a common framework for the physics of clocks in these different situations.
\end{abstract}

\section{Keywords}

Relativity, Time Dilation, Space-Time Ontology, Clock Slowdown, Inertial Clocks, Absolute Space and Time, Inertial Mass, Covariance Principle, Ideal Clocks

\section{Introduction}

Numerous 20th century physics experiments proved beyond doubt that clock measurements vary based on clock position and velocity in a local gravitational field. The global positioning system (GPS) is based upon these facts. Interestingly, despite the reality of the local gravitational fields, the theory that deals with velocity-based clock changes (special relativity) [1] ignores local gravity, assuming that it has no relevance. General relativity, on the other hand, is based on a metric representation of gravity in its treatment of clock changes [2]. Both theories focus on ideal clocks in space and time and ignore inertial mass contributions to the phenomena. Our modern world operates on the GPS that combines velocity and position in the local gravitational field. These are almost never treated together in special relativity texts, due to the imagined absence of gravity in special relativity theory.

The best-known clock experiments are Hafele-Keating [3], which treats clocks in relative motion in a local gravitational field; and Pound-Rebka [4], which 
treats clocks displaced relative to local gravitational field. In both instances, local gravity establishes the preferred local absolute coordinate system, with the origin in both cases being the local center-of-mass. In special theory moving clocks slow down and there exists no preferred frame; however in order to interpret the fact that clocks speed up or slow down according to whether they move East or West, one must define a preferred frame at the center-of-mass. These facts demand treatment based on local absolute coordinates and that is the goal of this paper.

The plan of this paper is as follows.

Section 1, the Introduction, summarizes the 20th century consensus that "time dilation" occurs and is physically real, with one aspect attributed to special relativity and another to general relativity.

Section 2 provides a novel overview of ideal clocks versus real inertial clocks.

Section 3 provides relevant background and context from 21st-century experimental physics, and formulates a novel physical, rather than mathematical, basis for the Minkowski invariance.

Section 4 develops a geometric algebra formulation of invariance following Hestenes. After relating his multi-vector to our photon model of invariance, we derive a relation based upon the fact that measurements of clocks in relative motion vary in such a way that an invariance relation is preserved across frames in relative motion. Unlike other derivations of this relation, ours is based upon the assumption of absolute space and time. Apparent differences in time are given a novel interpretation.

Section 5, physics in absolute space and time, differs from Hestenes' derivation that is based on Lorentz transformation. Our derivation of physics in absolute space yields the classical Hamiltonian that is generally believed to be based on relativistic space-time. This is a significant novel result that forms the basis for the inertial analysis of clocks in motion.

Section 6, the physics of inertial clocks, introduces the idea of real physical clocks as opposed to the "ideal" clocks of relativity. It lays the groundwork for the physics of the following sections.

Section 7 on time measurement analyzes the basis of atomic clocks. It is a review and summary of the physics underlying atomic clocks. There is little new physics in the section but it provides details that show the commonality of the simple harmonic oscillator approach for inertial clocks.

Section 8 analyzes clocks in relative motion, derives the key result of this paper, based on the covariance principle of physics applied to identical clocks in separate coordinate frames. This result does not apply to special relativity theory because it is based on kinetic energy difference between the accelerated clock and the stationary clock, and the effect on inertial mass due to the equivalent mass of this energy. In special relativity there is no preferred frame and the mass in question is reset to rest mass in every frame, preventing the physical basis of our analysis, i.e. the difference in kinetic energy of the two inertial clocks. In our theory local absolute space defines the preferred frame; the novel physics is 
straightforward, yielding exactly the clock behavior determined by 20th century physics.

Section 9 discusses the rationale used earlier to justify the derivation of the invariance relation, and clarifies the rationale based on the physics results just derived. It then applies this reasoning to the Hafele-Keating experiment. The actual results support our theory in absolute space and time, and it is necessary to depart from special relativity to explain these experimental results.

Section 10 treats positional dependence in the local absolute frame. It derives the formula for inertial clocks based on our energy theory that interprets both kinetic energy and gravitational energy in the same context. This interpretation differs from the generally unrelated interpretations of clock behavior in special and general relativity texts, but physical results are the same.

Section 11 discusses analysis of classical and quantum clocks in the context of a 2020 paper. The assumptions underlying the quantum treatment of clocks are similar to our assumptions concerning energy and absolute time and space, and differ from the Lorentz-based assumptions of special relativity.

Section 12 provides a summary of the paper.

\section{Overview Inertial Clocks versus Ideal Clocks}

Ideal clocks operate in a geometric universe; real clocks operate in a physical universe with inertial mass. The geometric model rotates time into space via Lorentz transformation on coordinates, while inertial clock models bring the relativistic $\gamma(v, c)$-factor into the definition of inertial mass $m=\gamma m_{0}$ and that is the extent of which $\gamma$ enters the physics. Length contraction and velocity addition do not appear in energy-time theory. Newtonian dynamics based on $F=m a$ show an inverse relation between inertial mass $m$ and acceleration $a$ for constant force $F$. Physically this exhibits the fact that inertia resists acceleration and hence clocks that have been accelerated in absolute space slow-down. Time plays no variable role in this dynamic; universal time is the same everywhere in the universe; it does not "flow differently" with local circumstances. One key principle in operation is conservation of energy and periodic transfer of energy associated with disturbances in the media. Another key principle is the covariance principle: Physics must not change, regardless of change in coordinate-based formalism. The time-based dependence of thermodynamic systems is seen in the $\gamma(v, c)$ function across all inertial clocks, based primarily upon the covariance of physics across frames in relative motion.

\section{Relevant Background}

The fundamental theories of "clock change" attribute the change to geometric transformation; the Lorentz transformation in special relativity and generalized space-time transformations in general relativity, which is, in general, not space-time Lorentz transformable. In this sense the physics is somewhat compartmentalized, with "flat-space" geometry describing velocity-based clock change and "curved 
space-time" geometry describing position-based change. This is further complicated by the fundamental proposition that no coordinate system can affect physics. Therefore, to remove whatever confusion accompanies these geometric formulations, we desire physics-based, rather than geometry-based, formulation. To establish such we begin with the existence of photons as disturbances in a gravitational field.

A major event occurred in 2017 when both photons and gravitons were detected from inspiraling neutron stars [5]. After traveling through gravitational fields for 1 billion years, the photon-disturbance and graviton-disturbance arrived at earth within roughly a microsecond of each other, establishing for the first time the equivalence of the speed of light in a gravitational field and the speed of gravity waves in the same field.

Light waves derive from Maxwell's equations with the speed given by $c=1 / \sqrt{e_{0} \mu_{0}}$, while gravitational waves derive from Heaviside-Einstein linear equations based on Newton's gravitational constant $G$. As the waves propagate in the same medium, we relate $\varepsilon$ and $\mu$ to $G$ and derive Equation (1). Will [6] discusses the TH $\varepsilon \mu$ formalism relating $\varepsilon$ and $\mu$ to $G$.

$$
c=\frac{1}{\sqrt{\varepsilon_{0} \mu_{0}}} \Rightarrow[\varepsilon(G) \mu(G)]^{-1 / 2}=\left[\left(\frac{-1}{4 \pi G}\right)_{\varepsilon}\left(\frac{-4 \pi G}{c^{2}}\right)_{\mu}\right]^{-1 / 2}=\left(c^{-2}\right)^{-1 / 2}=c
$$

Based on this detection, we know that photons and gravitons obey propagation law $|\boldsymbol{x}|=c t$. It is this basic physical law that we wish to build upon, rather than upon geometry independent of material reality. To do so we describe change in position $\mathrm{d} x$ of the photon during a change in time, duration $\mathrm{d} t$, as given by $|\mathrm{d} x|=c \mathrm{~d} t$. A vector magnitude is equal to the scalar distance. Hestenes, in Geometric Calculus, defines an entity $X=c t+\boldsymbol{x}$ which is a multi-vector scalar travel distance and travel direction representing the photon in our development. An associated entity, $\tilde{X}=c t-\boldsymbol{x}$ represents an anti-photon, or photon moving in the opposite direction $(-\boldsymbol{X})$ for the same time $t$ as shown in Figure 1.

The position multi-vector does not relate space to time other than as a disturbance traversing space for a given time. As this disturbance in the field has energy and momentum, we develop an energy-time theory of clocks rather than a space-time theory. Our goal is to formulate a theory of inertial clock slowdown, compatible with both special and general relativity.

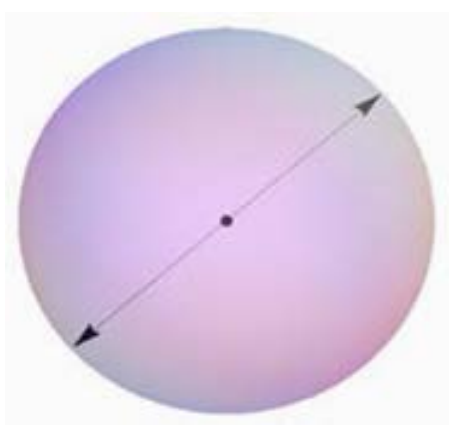

Figure 1. Photon propagation. 
Since we are dealing with coordinate systems we begin by recalling that no coordinate system can affect physics. Coordinates are names or labels of "points" in time and space and have no effect at all on physical reality. When a chosen coordinate system is applied to local reality, physical measurements performed are expressed in terms of changes detected in local time or space, $\mathrm{d} t$ or $\mathrm{d} \boldsymbol{x}$ respectively. Thus, had we chosen a different, "primed" coordinate frame, measurements would be expressed in terms of $\mathrm{d} t^{\prime}$ or $\mathrm{d} \boldsymbol{x}^{\prime}$ respectively.

Einstein's Principle of Covariance stipulates that laws of physics should be the same in all coordinate frames. Time and space are related in local absolute frames via one parameter, the speed of light $c$ such that $x= \pm c t,(\mathrm{~d} x= \pm c \mathrm{~d} t)$. With this absolute relation between time and space, we obtain two key physics relations with $c=1$ : distance and invariance:

$$
\begin{aligned}
& \mathrm{d} s^{2}=\mathrm{d} t^{2}+\mathrm{d} x^{2} \rightarrow \infty \quad \text { Pythagorean distance in 4-space, } \\
& \mathrm{d} s^{2}=\mathrm{d} t^{2}-\mathrm{d} x^{2}=0 \quad \text { Minkowski invariance in 4-space. }
\end{aligned}
$$

\section{Geometric Algebra Formulation of Invariance}

Following Hestenes [7], we define geometric algebra multi-vector $X=c t+\boldsymbol{x}$ $\Rightarrow \mathrm{d} X=c \mathrm{~d} t+\mathrm{d} \boldsymbol{x}$ and conjugate $\tilde{X}=c t-\boldsymbol{x} \Rightarrow \mathrm{d} \tilde{X}=c \mathrm{~d} t-\mathrm{d} \boldsymbol{x}$. From these it follows that

$$
X \tilde{X}=0 .
$$

and also that $X \tilde{X}^{\prime}=0$ when $X^{\prime}=c t^{\prime}+\boldsymbol{x}^{\prime}$. These invariance relations apply in all frames in which time-based distance is defined by the speed of light. For generality, we define invariance with respect to frames in relative motion at speeds slower than light. Consider one rest frame to have its origin at the local center-of-mass and a moving frame that moves with velocity $v$ with respect to the rest frame, such that $\boldsymbol{x}=\boldsymbol{v} t$. If we replace $\boldsymbol{x}$ in our $X$ multi-vector by $\boldsymbol{v} t$ we obtain $X=c t+v t$ and $\tilde{X}=c t-v t$. Unfortunately, this destroys invariance, since $X \tilde{X} \neq 0$ and $X \tilde{X}^{\prime} \neq 0$. However, invariance is such a powerful part of $20^{\text {th }}$ century physics we attempt to rescue the concept by declaring measurements performed in two different frames yield

$$
\frac{\mathrm{d}}{\mathrm{d} t}\left(X \tilde{X}-X^{\prime} \tilde{X}^{\prime}\right)=0
$$

If this is true, we have found an invariance relation relating two frames in relative motion - an absolute reference frame $S$ and a frame $S^{\prime}$ moving with respect to $S$. This will allow us to develop physics that is valid in either frame. The frames move with velocity $v$ and $\boldsymbol{v}^{\prime}$, respectively, so we proceed to differentiate Equation (3);

$$
\frac{\mathrm{d}}{\mathrm{d} t}\left[\left(c^{2}-v^{2}\right) t^{2}-\left(c^{2}-v^{\prime 2}\right) t^{\prime 2}\right]=0 \Rightarrow \frac{\mathrm{d}}{\mathrm{d} t}\left[\left(c^{2}-v^{2}\right) t^{2}\right]=\frac{\mathrm{d}}{\mathrm{d} t}\left[\left(c^{2}\right) \tau^{2}\right],
$$

where we have replaced $t^{\prime}$ by $\tau$ with no change of meaning, and we have arbitrarily set $\boldsymbol{v}^{\prime}=0$ (and adjusted $\boldsymbol{v}$ if necessary). Thus $\boldsymbol{v}$ is the relative velocity of the two frames, with primed frame $S^{\prime}$ moving with velocity $v$ with 
respect to the unprimed (absolute) frame $S$. Clearly $t \neq \tau$ and the relationship between $t$ and $\tau$ is a function of relative velocity $v ; t=\gamma(v) \tau$. A subtlety that may go unnoticed is that the invariance in question is not a geometric relation; it is in support of covariance of physics equations, where the variables represent measurements. The "time durations" $\mathrm{d} t$ and $\mathrm{d} t$ ' represent clock readings in the unprimed and primed coordinate frames respectively. For example, at 1:41:20 in his Stanford video on special relativity [8] Leonard Susskind asks: "what is this quantity $t$ '?" He answers: "It's the reading of a moving clock." That is also our answer: $t$ and $t^{\prime}$ represent clock readings (time measurements) in frames in relative motion. We complete the calculation and then interpret the result.

$$
2 t\left(c^{2}-v^{2}\right)=\left(c^{2}\right) 2 \tau \frac{\mathrm{d} \tau}{\mathrm{d} t} \Rightarrow\left(1-\frac{v^{2}}{c^{2}}\right)=\frac{\tau}{t} \frac{\mathrm{d} \tau}{\mathrm{d} t} \Rightarrow \frac{1}{\gamma} \frac{\mathrm{d} \tau}{\mathrm{d} t} \Rightarrow \frac{1}{\gamma^{2}}
$$

Thus

$$
\gamma(v)=\frac{1}{\sqrt{1-v^{2} / c^{2}}} \text { for constant } v \neq v(t) .
$$

If absolute time $t$ is measured by a clock in the absolute frame, then apparent time $\tau$, associated with the moving frame, will be that measured by an identical clock in the moving frame $S^{\prime}$. Thus we may view $\gamma(v)$ as a clock calibration factor that must be satisfied for our invariance to hold true for all moving frames. To know that our clocks are "identical", we compare them at rest in the preferred frame, and then accelerate them to velocity $v$ of the target frame. The acceleration of clocks is undetermined in relativity, but the added kinetic energy is equivalent to added mass and hence the inertia of the clocks will change. To analyze this we develop physics based on the invariance relations we have found.

\section{Physics in Absolute Time and Space}

The unprimed system $S$ is considered to be at rest in the local absolute framework while the primed system is moving with velocity $\boldsymbol{v}=\dot{\boldsymbol{x}}$ in the rest frame. From Equation (2) and Equation (3) we obtain $(\Delta X)(\Delta \tilde{X})=c^{2}(\Delta t)^{2}-(\Delta x)^{2}$ and $\left(\Delta X^{\prime}\right)\left(\Delta \tilde{X}^{\prime}\right)=c^{2}(\Delta \tau)^{2}-\left(\Delta \boldsymbol{x}^{\prime}\right)^{2}$. Let $\Delta \boldsymbol{x}=\boldsymbol{v} \Delta t$ and $\Delta \boldsymbol{x}^{\prime}=\boldsymbol{v}^{\prime} \Delta \tau$. If invariance is preserved, and we set $\boldsymbol{v}^{\prime}=0$ we obtain Hestenes' relation:

$$
\left(\Delta X^{\prime}\right)\left(\Delta \tilde{X}^{\prime}\right)=c^{2}(\Delta \tau)^{2}=(\Delta X)(\Delta \tilde{X}) .
$$

Divide both sides of the equation by $\Delta \tau$ twice, yielding

$$
\left(\frac{\Delta X}{\Delta \tau}\right)\left(\frac{\Delta \tilde{X}}{\Delta \tau}\right)=c^{2}
$$

If $\Delta \tau \rightarrow \mathrm{d} \tau$ then four-velocity $V$ is the entity $(\mathrm{d} X / \mathrm{d} \tau)$ with conjugate $\tilde{V}=\mathrm{d} \tilde{X} / \mathrm{d} \tau$, hence

$$
V \tilde{V}=\left(\frac{\mathrm{d} X}{\mathrm{~d} \tau}\right)\left(\frac{\mathrm{d} \tilde{X}}{\mathrm{~d} \tau}\right)=c^{2}
$$


As Hestenes observes: "Unlike three-velocities, the four-velocity has a constant magnitude independent of the particle history." The Minkowski invariance is key. Though Hestenes derives his relations from the Lorentz transformation, we have not, and will not, invoke Lorentz herein, because it does not carry through to the gravitational aspects of clocks.

Recall that $X(t)=c t+\boldsymbol{x}(t), \tilde{X}(t)=c t-\boldsymbol{x}(t)$, therefore

$$
\frac{\mathrm{d} X}{\mathrm{~d} t}=c+\frac{\mathrm{d} \boldsymbol{x}}{\mathrm{d} t}=c+\boldsymbol{v} .
$$

If moving clock time $\tau$ is used, and $X(\tau)=c t(\tau)+x(\tau)$ then

$$
\frac{\mathrm{d} X}{\mathrm{~d} \tau}=\frac{\mathrm{d} t}{\mathrm{~d} \tau}\left(c+\frac{\mathrm{d} \boldsymbol{x}}{\mathrm{d} t}\right)=\gamma(c+\boldsymbol{v})
$$

since $t=\gamma \tau$ then $\mathrm{d} t=\gamma \mathrm{d} \tau \Rightarrow \gamma=\mathrm{d} t / \mathrm{d} \tau$. Although the above development appears geometric, we stipulate that physics involves inertial mass; therefore we introduce mass into the development. We do so with the right-hand terms of Equation (11) and multiply these by the rest mass $m_{0}$ of a particle, to obtain $m_{0} \gamma(c+v)$. The four-vector has the form scalar plus vector, with $\gamma m_{0} c=$ scalar and $\gamma m_{0} \boldsymbol{v}=$ vector. Since $\gamma$ is dimensionless $\gamma m_{0} \boldsymbol{v}$ has units of momentum:

$$
\boldsymbol{p}=m \boldsymbol{v} .
$$

If this is to make sense, we define the inertial mass $m$ to be

$$
m \equiv \gamma m_{0}
$$

implying that inertial mass increases with velocity, yielding rest mass at $\boldsymbol{v}=0$. This relation has been confirmed countless times in twentieth century particle physics. [9] Thus $P=m_{0} V$. The scalar $\gamma m_{0} c$ has units of momentum, but no obvious interpretation. If we multiply and divide by $c$ we obtain $\gamma m_{0} c^{2} / c$. Since $\gamma m_{0} c^{2}$ has units of energy, we define $E=\gamma m_{0} c^{2}$ as particle energy. Fourmomentum $P=m_{0} V=\left(\frac{E}{c}+\boldsymbol{p}\right)$ implies a conjugate $\tilde{P}=m_{0} \tilde{V}=\left(\frac{E}{c}-\boldsymbol{p}\right)$ thus $P \tilde{P}=\frac{E^{2}}{c^{2}}-p^{2}$ while Equation (9) shows that

$$
P \tilde{P}=m_{0} V m_{0} \tilde{V}=m_{0}^{2} V \tilde{V}=m_{0}^{2} c^{2}
$$

hence

$$
\frac{E^{2}}{c^{2}}-\boldsymbol{p}^{2}=m_{0}^{2} c^{2}
$$

We now have a four-momentum invariance relation based on scalar $E$ plus 3-vector momentum $\boldsymbol{p}$. This dynamical energy-momentum relation is the essence of particle physics. The rest mass is found by setting $\boldsymbol{p}=0 \Rightarrow E(0)=m_{0} c^{2}$. The energy expression $E(\boldsymbol{p})=\left(m_{0}^{2} c^{4}+c^{2} \boldsymbol{p}^{2}\right)^{1 / 2}$ implies the classical Hamiltonian [10]

$$
H(\boldsymbol{p})=E(\boldsymbol{p})=\left(m_{0}^{2} c^{4}+c^{2} \boldsymbol{p}^{2}\right)^{1 / 2} .
$$

This key invariance relation in physics, the Hamiltonian, was derived based 
on local absolute space and universal time. A Legendre transformation allows us to transform a function of some variable into another function of another variable without losing any information. Classical physics can be formulated in terms of Lagrangian $L=T-V$ where $T$ is kinetic energy and $V$ is potential energy. Application of Legendre transform to a Lagrangian produces a Hamiltonian $H=T+V$. Legendre transformations do not lose any information; they are self-inverse. So in going from Lagrangian to Hamiltonian we lose no information; a second application of Legendre, this time to the Hamiltonian, will restore the original information, in the form of the Lagrangian. Thus our Hamiltonian derived from absolute time and space should transform into the appropriate Lagrangian formulation. In Hamiltonian $H=T+V$ for a free particle $(V=0)$ only the kinetic energy, $T=m_{0} v^{2} / 2$, is used. The total energy scalar defined above is $E=\gamma m_{0} c^{2}$. For velocities much less than the speed of light we expand total energy as

$$
E=\gamma m_{0} c^{2}=m_{0} c^{2}\left(1-\frac{v^{2}}{c^{2}}\right)^{-1 / 2} \cong m_{0} c^{2}+m_{0} v^{2} / 2
$$

Ignoring rest energy $m_{0} c^{2}$ the free particle kinetic energy is $T=m_{0} v^{2} / 2$, hence our absolute time and space Hamiltonian maps into classical non-relativistic mechanics as required.

\section{The Physics of Inertial Clocks}

We required only that clocks be constructed identically, but we never specified physically real clock construction. Clocks are sometimes conceptualized as time transducers but physical transducers such as piezoelectric devices convert mechanical stress into voltage, and voltage into mechanical stress. There exists no time transducer that converts time into something else that can be measured. All clocks count cycles; hence constructing a clock depends on designing a device that cycles with stable period. All useful time-measurement devices consist of multiple particles or subsystems and thus are subject to thermodynamic considerations [11] where:

"Thermodynamics deals with matter and interactions between matter."

Multiple subsystems are typically partitioned into a system of interest and its surroundings, and "Work refers to an interchange between a system and its surroundings." Our clocks are material in nature, hence inertial, and considered as non-intrusive entities present at the origin of the inertial reference frame of interest, or wherever else it is desirable to measure time in said frame.

\section{Time Measurement: The Diatomic Molecule Vibrational states}

Thermodynamic systems are modeled as subsystems that exchange energy within themselves and with their surroundings. Atomic clocks provide accurate time measurement devices, so we begin by modeling the vibrational state of a diatomic molecule, consisting of nuclear masses $m_{1}$ and $m_{2}$ and several elec- 
trons. Our partitioning framework involves exchange interactions between all charged particles. The fact that low mass electrons quickly adjust to any change of position of the relatively massive, slow-moving nuclei suggests that we partition the system into two nuclei embedded in a distributed charge environment [12] as seen in Figure 2.

Instantaneous actions are based on force exchange; it is convenient to interpret an integral over forces: $\boldsymbol{F}=-\boldsymbol{\nabla} \phi, \phi=\int \boldsymbol{F} \cdot \mathrm{d} \boldsymbol{x}$, where $\phi$ is the relevant potential. The diatomic molecule's nuclei are a distance $r$ apart and are surrounded by several electrons. The force of electric interaction is $\approx q_{1} q_{2} / r_{12}^{2}$ for the nuclei and $\approx q_{1} q_{e} / r_{1 e}^{2}$ between nucleus $q_{1}$ and a $q_{e}$ electron, $r_{1 e}$ distant. The Schrödinger equation potential energy is made up of electron-electron, electron-nucleus, and nucleus-nucleus coulomb interactions obtaining a set of electronic energy levels dependent on $r$, denoted by $u_{e}(r)$. If the masses are assumed constant, the nuclear mass $m_{n}=m_{1}=m_{2}$ is many thousands of times greater than electron mass $m_{e}$ so velocity $v_{e}$ of the electron is many times greater than the nuclear velocity. Figure 3 represents a relatively stationary nucleus in the cloud of fast-moving electrons which also interact with themselves. If all electrons were positioned between the two nuclei the positively charged nuclei would be attracted toward the center. If no electrons were located between the nuclei, the nuclei would force each other apart. The Born-Oppenheimer approximation considers the nuclei fixed, while studying the electrons in motion. For nuclei fixed a distance $r$ apart, the potential energy is made up of the sum of all coulomb interactions. Let the dependence of the ground state electronic energy on internuclear distance be $u_{e}(r)$, with typical form shown in Figure 3.

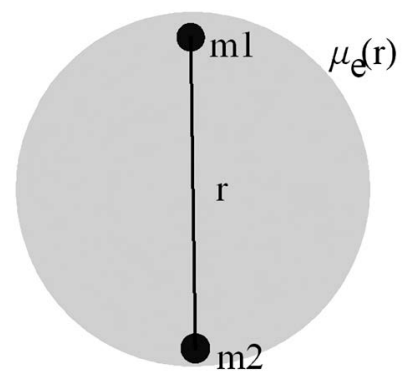

Figure 2. Diatomic molecule.

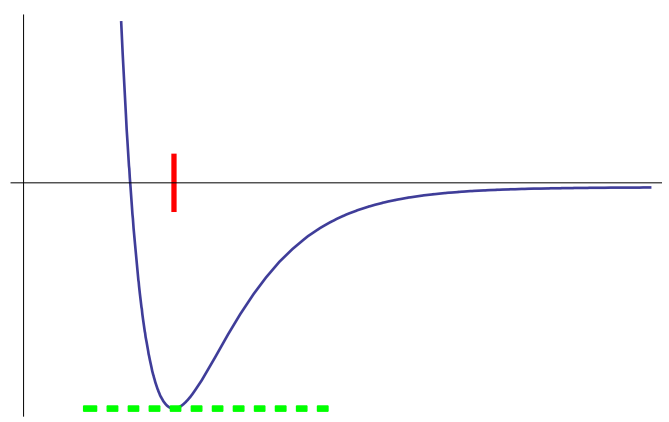

Figure 3. Ground state: electron energy $\mu_{\varepsilon}(r)$. 
Following Hill, we choose coordinates $x, y, z, r, \theta, \phi$ where $x, y, z$ refers to the position of the center of mass, $r$ is a separation on an axis $\xi$ connecting the two nuclei, $m_{1}$ and $m_{2}$, and $\theta$ and $\phi$ describe the orientation of axis $\xi$, associated with vibration of the nuclei along $\xi$ and the rotation of $\xi$ about $z$. Our interest is in the vibrational states; we restrict ourselves to small vibrations near the bottom of the potential well. If the ground state electronic energy is a stable configuration, the nuclei effectively exist in stable surroundings, the diatomic molecule is partitioned into a system of two nuclei in a surrounding electron "bath", labeled $u_{e}(r)$. We can investigate the (relatively slow) motion of the two nuclei in the coordinate system $x, y, z, r, \theta, \phi$ on the axis $\xi$ connecting the two nuclei, $m_{1}$ and $m_{2}$; and $\theta$ and $\phi$ describe the orientation of axis $\xi$, as seen in Figure 4. Thus, in addition to the electron energy states, there exist energy states associated with vibration of the nuclei along $\xi$ and the rotation of $\xi$ about $z$. As our interest is in the vibrational states, it is customary to replace the actual $u_{e}(r)$ with a parabola which fits $u_{e}(r)$ in the neighborhood of its minimum, since, if a stable physical configuration exists, the state exists at a local minimum or ground energy. The Taylor series expansion around the local minimum at $r_{0}$ is

$$
u_{e}(r)=u_{e}\left(r_{0}\right)+\frac{\mathrm{d} u_{e}}{\mathrm{~d} r}\left(r-r_{0}\right)+\frac{1}{2} \frac{\mathrm{d}^{2} u_{e}}{\mathrm{~d} r^{2}}\left(r-r_{0}\right)^{2}+\cdots
$$

The linear term vanishes near the minimum since $\left.\frac{\mathrm{d} u_{e}}{\mathrm{~d} r}\right|_{r=r_{0}}=0$ so if $\Delta u_{e}(r)=u_{e}(r)-u_{e}\left(r_{0}\right)$ then

$$
\Delta u_{e}(r)=\left.\frac{1}{2} \frac{\mathrm{d}^{2} u_{e}}{\mathrm{~d} r^{2}}\right|_{r=r_{0}}\left(r-r_{0}\right)^{2} .
$$

The energy change $\Delta u_{e}$ represents work done on, or by, the system. Following Hill [13], the inter-nuclear distance is $r=\xi_{2}-\xi_{1}$, where the line between the center of the two nuclei is the $\xi$-axis with arbitrary origin and $\xi_{2}$ (location of nuclear mass $\left.m_{2}\right)>\xi_{1}$.

$$
u_{e}=\frac{k}{2}\left(\xi_{2}-\xi_{1}-r_{0}\right)^{2}
$$



Figure 4. Vibration axis. 
Newton's equation of motion is $\boldsymbol{f}=m a$; force is the derivative of the potential $f=-\nabla u$, hence

$$
\begin{array}{r}
m_{1} \ddot{\xi}_{1}=-\frac{\mathrm{d} u_{e}}{\mathrm{~d} \xi_{1}}=k\left(\xi_{2}-\xi_{1}-r_{0}\right) \\
m_{2} \ddot{\xi}_{2}=-\frac{\mathrm{d} u_{e}}{\mathrm{~d} \xi_{2}}=k\left(\xi_{2}-\xi_{1}-r_{0}\right)
\end{array}
$$

Multiply Equation (21) by $-m_{2}$, Equation (22) by $m_{1}$, add the equations, and divide by $m_{1}+m_{2}$ to obtain

$$
\mu \ddot{x}=-k x
$$

where $x=r-r_{0}$ and $\mu=\frac{m_{1} m_{2}}{m_{1}+m_{2}}$. Equation (23) is the equation of motion of the 1D harmonic oscillator with "reduced" mass $\mu$. The cycle frequency of the classical motion is $2 \pi v=\sqrt{k / \mu}$, but what exactly is cycling? In literally every clock system, energy cycles through two conjugate modes of the system, capable of at least temporary storage of energy; typically kinetic energy of the system exchanged with potential energy of the environment that the system is linked to; inherently thermodynamic. If potential energy is considered zero at $r=r_{0}$ then kinetic energy is maximum at $r_{0}$. As $r$ decreases, the ions come closer together and repel each other more strongly, slowing down the ions according to $\mathrm{d} \boldsymbol{p} / \mathrm{d} t=-k \boldsymbol{x}$ where $\boldsymbol{F}=\mathrm{d} \boldsymbol{p} / \mathrm{d} t=-\nabla\left(k x^{2} / 2\right)$. When $\quad x=r-r_{0} \quad$ is negative $\left(r<r_{0}\right)$ the change in momentum is positive, forcing the ions apart from each other. When $x$ is positive, the ions are far apart and the electron cloud attracts both more strongly. A parabola that approximates the potential minimum is symmetric, changing the sign of the force when $r$ crosses $r_{0}$, reversing the cycle of motion. If $x \sim \sin \omega t$ where $\omega$ is the circular frequency $\omega=2 \pi / \Delta T$ then $x=\sin \omega t, \quad \dot{x}=\omega \cos \omega t$, and $\ddot{x}=-\omega^{2} \sin \omega t$ hence

$$
\ddot{x}+\omega^{2} x=0 \text {. }
$$

Generally speaking, every system capable of vibrating around a minimum potential energy (an approximate parabolic shape) will oscillate as a sine wave function of time plus a phase. It is the act-of-counting oscillation cycles that measures time. From our derivation of absolute physics we have $\boldsymbol{p}=m \boldsymbol{v}$ hence $\dot{\boldsymbol{p}}=m \ddot{\boldsymbol{x}}=-k \boldsymbol{x}$, thus

$$
\ddot{x}+(k / m) x=0 \Rightarrow \omega^{2}=k / m, \omega=\sqrt{k / m}
$$

The ions move slowly compared to the surrounding electrons so the oscillating speed of the ions is nonrelativistic. This motion is internal to the clock mechanism, which may itself be moving with velocity $v$ with respect to our rest frame. We analyze this clock motion next.

\section{Analysis of Clocks in Relative Motion: The Covariance Principle}

The only meaningful way to say that clocks tell the same time is to construct 
them identically in some place side-by-side and observe time measurements. This is readily done; vast numbers of clocks exist, all of which count cycles based on some appropriate oscillating mechanism. Assume that we have two identical clocks initially at rest. Twentieth century physics tells us that one of the clocks will slow down if the clocks are in uniform relative motion, but which clock? The answer is whichever clock is accelerated to velocity $v$ with respect to the other clock, which remains at rest. In other words, when we apply work or transfer kinetic energy to a clock, it slows down. We make use of the covariance of the physics equations in two separate frames, $S$ and $S^{\prime}$, where $S$ is the rest frame and $S^{\prime}$ is the frame that moves with velocity $v$ as measured in the $S$ frame, both shown in Figure 5. The equation of motion has the same form in both frames:

$$
\frac{\mathrm{d} \boldsymbol{p}}{\mathrm{d} t}=-k \boldsymbol{x}, \frac{\mathrm{d} \boldsymbol{p}^{\prime}}{\mathrm{d} t^{\prime}}=-k^{\prime} \boldsymbol{x}^{\prime}
$$

Newton's law $\boldsymbol{F}=\mathrm{d} \boldsymbol{p} / \mathrm{d} t$ for the clock at rest has restoring force on the sprung mass $\boldsymbol{F}=-k \Delta \boldsymbol{x}$. We assign an equivalent relation $\boldsymbol{F}^{\prime}=\mathrm{d} \boldsymbol{p}^{\prime} / \mathrm{d} t^{\prime}=-k^{\prime} x^{\prime}$ to the clock in motion, where $x^{\prime}=\Delta x$ is the stretch of the spring and we assume $k^{\prime}=k$. Velocity $v_{0}$ of the rest clock is zero in absolute space, while the velocity of sprung mass $m_{0}$ of the rest clock is $\boldsymbol{u}=\mathrm{d} \boldsymbol{x} / \mathrm{d} t=\dot{\boldsymbol{x}}$. The moving clock is assumed to be initially at rest, and then to be accelerated to velocity $v$, so the velocity of the sprung mass $m^{\prime}$ of the moving clock is $v+\boldsymbol{u}^{\prime}$ in absolute space with $\boldsymbol{v}=\mathrm{d} \boldsymbol{x} / \mathrm{d} t$ and $\boldsymbol{u}^{\prime}=\mathrm{d} \boldsymbol{x}^{\prime} / \mathrm{d} t=\dot{\boldsymbol{x}}^{\prime}$. In these terms the momentum of the sprung mass of the rest clock is given by $\boldsymbol{p}=m_{0}\left(\boldsymbol{v}_{0}+\boldsymbol{u}\right)$ while the momentum of the sprung mass of the moving clock is $\boldsymbol{p}^{\prime}=m^{\prime}\left(\boldsymbol{v}+\boldsymbol{u}^{\prime}\right)$. We write equations of motion of such mass in both unprimed (rest) and primed (moving) frames:

$$
\frac{\mathrm{d} \boldsymbol{p}}{\mathrm{d} t}=m_{0} \frac{\mathrm{d} \boldsymbol{u}}{\mathrm{d} t}=m_{0} \ddot{\boldsymbol{x}}=-k \boldsymbol{x}, \frac{\mathrm{d} \boldsymbol{p}^{\prime}}{\mathrm{d} t^{\prime}}=m^{\prime} \frac{\mathrm{d}}{\mathrm{d} t^{\prime}}\left(\boldsymbol{v}+\boldsymbol{u}^{\prime}\right)=-k \boldsymbol{x}^{\prime}
$$

SO

$$
\frac{\mathrm{d}}{\mathrm{d} t^{\prime}}=\frac{\mathrm{d}}{\mathrm{d} t / \gamma}=\gamma \frac{\mathrm{d}}{\mathrm{d} t} \Rightarrow \frac{\mathrm{d} \boldsymbol{p}^{\prime}}{\mathrm{d} t^{\prime}}=m^{\prime} \frac{\mathrm{d}}{\mathrm{d} t^{\prime}}\left(\boldsymbol{v}+\boldsymbol{u}^{\prime}\right)=\gamma m^{\prime} \frac{\mathrm{d} \boldsymbol{u}^{\prime}}{\mathrm{d} t}=-k \boldsymbol{x}^{\prime}
$$

\section{Rest Clock}
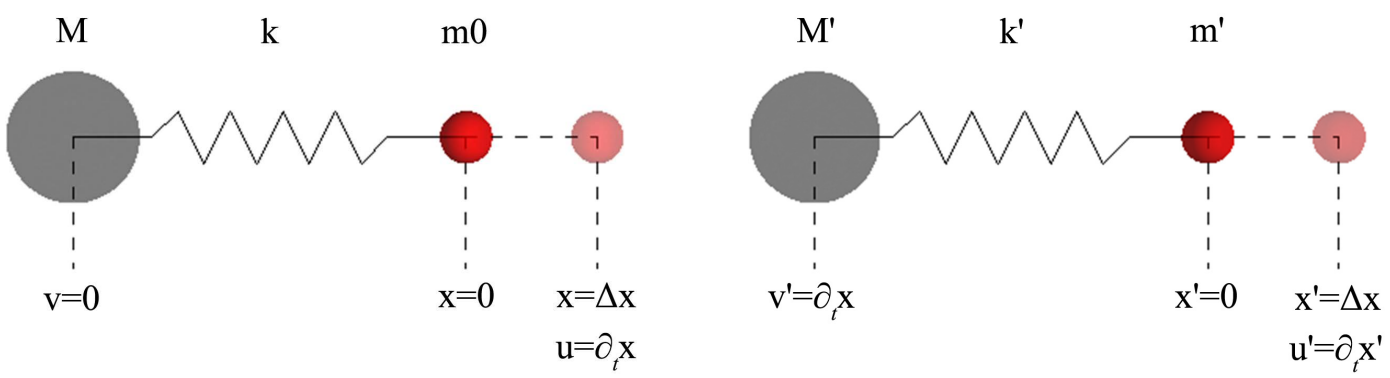

Figure 5. Diagrams with relevant parameters for rest and moving clocks. 
Since $\boldsymbol{u}^{\prime}=\frac{\mathrm{d} \boldsymbol{x}^{\prime}}{\mathrm{d} t}$ and $m^{\prime}=\gamma m_{0}$ we have $\frac{\mathrm{d} \boldsymbol{p}^{\prime}}{\mathrm{d} t^{\prime}}=\gamma m^{\prime} \frac{\mathrm{d}^{2} \boldsymbol{x}^{\prime}}{\mathrm{d} t^{2}}=-k \boldsymbol{x}^{\prime}$ $\Rightarrow \gamma^{2} m_{0} \ddot{\boldsymbol{x}}^{\prime}=-k \boldsymbol{x}^{\prime}$.

Summarizing:

$$
\begin{gathered}
\frac{\mathrm{d} \boldsymbol{p}^{\prime}}{\mathrm{d} t^{\prime}} \Rightarrow \frac{\mathrm{d}^{2} \boldsymbol{x}^{\prime}}{\mathrm{d} t^{2}}+\left(\frac{\omega_{0}}{\gamma}\right)^{2} \boldsymbol{x}^{\prime}=0 \Rightarrow \ddot{\boldsymbol{x}}^{\prime}+\omega^{\prime 2} \boldsymbol{x}^{\prime}=0 \\
\frac{\mathrm{d} \boldsymbol{p}}{\mathrm{d} t} \Rightarrow \frac{\mathrm{d}^{2} \boldsymbol{x}}{\mathrm{d} t^{2}}+\omega_{0}^{2} \boldsymbol{x}=0 \Rightarrow \ddot{\boldsymbol{x}}+\omega_{0}^{2} \boldsymbol{x}=0
\end{gathered}
$$

In terms of universal time $t$, the equation of motion of the rest clock yields frequency $\omega_{0}$, while the frequency of the moving clock yields $\omega^{\prime}=\omega_{0} / \gamma$. This establishes time dilation for inertial clocks in relative motion in universal time and space.

Should one become confused over $\boldsymbol{x}$ and $\boldsymbol{x}^{\prime}$ above, the equations of motion for sprung-mass treat $\boldsymbol{x}$ and $\boldsymbol{x}^{\prime}$ as the position variables for $m$ and $m^{\prime}$ respectively, $\boldsymbol{x}$ can be replaced by $\boldsymbol{x}_{m}$ and $\boldsymbol{x}^{\prime}$ be replaced by $\boldsymbol{x}_{m^{\prime}}^{\prime}$ such that the relevant velocities are denoted by:

$$
\boldsymbol{u}=\frac{\mathrm{d} \boldsymbol{x}}{\mathrm{d} t} \equiv \frac{\mathrm{d} \boldsymbol{x}_{m}}{\mathrm{~d} t} \text { and } \boldsymbol{u}^{\prime}=\frac{\mathrm{d} \boldsymbol{x}^{\prime}}{\mathrm{d} t} \equiv \frac{\mathrm{d} \boldsymbol{x}_{m^{\prime}}^{\prime}}{\mathrm{d} t}
$$

and every step can be carried out using these explicit mass-based position coordinates. Of course one obtains the same final results. Equation (29) and Equation (30) are formulated in terms of universal time, $t$, as should be expected in a theory of absolute time and space. The frequency $\omega^{\prime}$ of the moving oscillator is less than rest frequency $\omega_{0}$ since $\gamma>1$. Frequency $\omega_{0}$ is inversely related to the rest clock period: $\omega_{0} \sim 1 / \Delta t$ and frequency $\omega^{\prime}$ of the moving oscillator is inversely related to the period of the moving clock $\omega^{\prime} \sim 1 / \Delta t^{\prime}$. From these we obtain $\frac{\omega_{0}}{\omega^{\prime}}=\gamma=\frac{\Delta t^{\prime}}{\Delta t}$. Since clocks tell time by counting periods let us assume an absolute time period $\Delta T$ is measured by each clock. If the rest clock counts $n$ periods $(\Delta t)$ during $\Delta T$ and the moving clock counts $n^{\prime}$ periods $\left(\Delta t^{\prime}=\Delta \tau\right)$ then we obtain

$$
\Delta T=n \Delta t=n^{\prime} \Delta t^{\prime}
$$

where $n$ and $n^{\prime}$ are the respective readings of the clocks in $S$ and $S^{\prime}$. From Equation (32) we derive

$$
\frac{n}{n^{\prime}}=\frac{\Delta t^{\prime}}{\Delta t}=\gamma
$$

hence $n=\gamma n^{\prime}$. Thus the clock moving with velocity $v$ runs more slowly by a factor equal to the inertial factor $\gamma$. This clock slowdown agrees with relativistic time dilation but is based on absolute time. For instance, the derivative $\mathrm{d} / \mathrm{d} t^{\prime}$ refers to the change in something with respect to the time measured in the moving system. Thus $\mathrm{d} t^{\prime}$ represents time measured in $S^{\prime}$ and $\mathrm{d} t$ represents time measured in rest frame $S$. But these times are clock readings $n$ and $n^{\prime}$, hence $n \sim \mathrm{d} t$ and $n^{\prime} \sim \mathrm{d} t^{\prime}$ and we obtain 


$$
\frac{n}{n^{\prime}}=\frac{\mathrm{d} t}{\mathrm{~d} t^{\prime}}=\gamma
$$

These readings are multiples of the clock tick or oscillation periods, denoted by $\Delta t$ and $\Delta t^{\prime}$ respectively for rest and moving frames. These terms are inverse to the respective frequencies: $\omega_{0} \sim 1 / \Delta t, \omega^{\prime} \sim 1 / \Delta t^{\prime}$. In terms of clock periods, the relations appear reversed: $\frac{\Delta t^{\prime}}{\Delta t}=\gamma$. So do not confuse clock reading $\mathrm{d} t$ with rest clock period $\Delta t$, or $\mathrm{d} t^{\prime}$ with $\Delta t^{\prime}$.

For a spring-based clock $f=-k \boldsymbol{x}$ the frequency of oscillation of the moving clock is $\omega^{\prime}=\omega_{0} / \gamma$ thus the moving clock "runs slower" than the clock at rest. The increase in inertial mass is the equivalent mass of the kinetic energy of the moving clock gained when the moving clock was accelerated from rest to velocity $v$ in the absolute frame. The key fact is that:

Our analysis of inertial clocks leads to exactly $\omega^{\prime}=\omega_{0} / \gamma \Rightarrow \mathrm{d} t / \mathrm{d} \tau=\gamma$ the clock relationship that was experimentally confirmed in the twentieth century.

\section{9. "Calibration" vs. Thermodynamics}

In our absolute time and space derivation of invariance leading to the Hamiltonian, the rest frame clock measures universal time $t$ while the moving clock measurement is $t^{\prime}$. Invariance between frames is preserved if clocks yield $\mathrm{d} t=\gamma(v) \mathrm{d} t^{\prime}$ with $\gamma(v)=\left(1-v^{2} / c^{2}\right)^{-1 / 2}$. How can one calibrate two clocks in this way? We cannot construct both clocks in the rest frame such that they can be calibrated for all mutual velocity $v$. Clocks must be constructed identically; there is no way to build such calibration into the moving clock since we choose which clock is to remain at rest and which is to be accelerated to velocity $v$. Yet while a calibration perspective fails, the thermodynamics of clock oscillators yields exactly the required $\gamma(v)$ relation, regardless of which clock accelerates from rest.

In deriving physics from the invariance relation, we sought to justify the invariance itself, in order to be able to apply the invariance if the $\gamma$ constraint was met. Application of the invariance, combined with mass, yielded $\gamma(v)$ such that $m=\gamma m_{0}$ with $\boldsymbol{p}=m \boldsymbol{v}$ and $E=m c^{2}$. This relation applies to all inertial mass and therefore to all harmonic oscillators, and hence all clocks. Thus clocks are effectively self-calibrating for all velocities, exactly preserving the invariance relation that yields the energy Hamiltonian.

In energy-time theory, based on universal simultaneity and preferred local frame, space-time symmetry is thermodynamically unrealistic: In the real world, say Denver, a plane taking off in any direction is predicted by space-time symmetry to run slower than the clock at the Denver airport. Of course this does not happen. As Hafele and Keating first showed, clocks on planes taking off to the east slow down, while clocks on planes taking off to the west speed up. As the rotating earth is not an inertial reference frame, thermodynamic theory addresses reality. The west-to-east velocity of the Denver airport is approximately 500 miles per hour, so the clock at Denver airport has associated velocity and 
momentum and energy $\{E, \boldsymbol{p}\} \sim\left\{m c^{2}, m \boldsymbol{v}\right\}$ going west-to-east. When Bob takes off from Denver heading east, he adds to the clock's local absolute velocity; its inertial mass will increase, its resistance to acceleration will increase, and his clock will slow down. But when Alice heads out for San Francisco, she will add a velocity component east-to-west that subtracts from the local absolute west-to-east velocity and thus travels slower; so Alice's clock does not slow down as she flies west, it speeds up, exhibiting the effect of a preferred frame. That is, a preferred frame is required to predict clock slowdown vs. speedup.

\section{Positional Dependence in the Local Field}

Our above thermodynamic analysis is based not on space-time symmetry, but on inertial mass $m=m_{0} \gamma(v)$; inertial factor $\gamma(v)$ relates to inertial mass, not to space and time. We extend this relation to general relativity [14] as

$$
m=m_{0} \gamma(\dot{\boldsymbol{r}}) \mathrm{e}^{\phi(\boldsymbol{r})}
$$

Here the mass of the particle depends upon the kinetic energy in a local absolute gravitational field as well as its position-dependent energy within a gravitational field with potential $\phi$. This inertial relation explains clock-slowing, as known and practiced in GPS.

Our "gravity free" definition of inertial mass, $m=\gamma m_{0}$ was found to include the kinetic energy in addition to rest mass: $m=m_{0}\left(1-v^{2} / c^{2}\right)^{1 / 2}$. In a gravitational field the energy of a mass depends upon the Newtonian potential $\phi=G M / r$ so let us naïvely add the gravitational term $2 \phi / c^{2}$ to the kinetic energy term as follows

$$
m=m_{0}\left[1-\left(\frac{v^{2}}{c^{2}}+\frac{2 \phi}{c^{2}}\right)\right]^{-1 / 2} \Rightarrow m_{0}\left[1+\frac{1}{2} \frac{v^{2}}{c^{2}}+\frac{\phi}{c^{2}}\right]
$$

yielding inertial mass and associated energies:

$$
\underset{\text { total energy }}{m c^{2}}=\underset{\text { rest }}{m_{0} c^{2}}+\underset{\text { kinetic }}{m_{0} v^{2} / 2}+\underset{\text { gravitational }}{m_{0} \phi}
$$

This formulation demonstrates the dependence of inertial mass on velocity $\dot{\boldsymbol{r}}$ through the local gravitational field and on position $r$ in the local gravitational field. Our simple addition of the gravitational potential $\phi=G M / r$ is only intuitively justified, however Poisson and Will [13] derive the same result in orthodox fashion based on the action

$$
S=-m_{0} c^{2} \int \mathrm{d} \tau=\int L \mathrm{~d} t
$$

where $\mathrm{d} \tau$ is the proper time after clock slowdown (speedup) is considered, and the Lagrangian

$$
L=-m c \sqrt{-\eta_{\alpha \beta} \frac{\mathrm{d} r^{\alpha}}{\mathrm{d} t} \frac{\mathrm{d} r^{\beta}}{\mathrm{d} t}}
$$

is evaluated as

$$
L=-m c \sqrt{1-\frac{2 \phi}{c^{2}}-\frac{v^{2}}{c^{2}}}
$$


in which case

$$
L=-m_{0} c^{2}+m_{0} v^{2} / 2+m_{0} \phi
$$

As explained in Section 5, our energy-time Hamiltonian is completely compatible with the classical Lagrangian approach, therefore the Lagrangian in Equation (41) yields physics in agreement with Equation (37). We again see that the time-dependence of clocks follows from energy-based analysis rather than geometry.

\section{Analysis of Classical and Quantum Clocks}

The issue of inertial clocks is one of current interest: Paige, Plato, and Kim [15] analyze classical and non-classical time dilation for quantum clocks. Quantum mechanics assumes universal time, and their analysis starts from a Hamiltonian modified to account for the inertial mass of internal energy as well as kinetic energy. Thus their key assumptions agree with our treatment of inertial clocks, and their clocks are also assumed to be based on composite particles, bringing thermodynamic aspects into play. They, too, view energy and inertial mass as equivalent, and they utilize the same relativistic Hamiltonian that we derived herein. In this context their predicted classical clock behavior agrees with our analysis and with experiment.

For non-classical analysis, quantum aspects such as the Uncertainty Principle complicate the picture: with momentum boosts it becomes impossible to define the exact position of the origin of the frame. Purely quantum aspects such as superposition, entanglement also complicate issues, but predicted differences are too small to measure with shifts about $10^{-10}$ smaller than observed.

The analysis of quantum clocks shares our key assumptions: universal time, relativistic Hamiltonian, and inertial mass. Their results show that clocks run slower by the inverse factor of $\gamma$. Of major significance: their analysis, like our analysis, is based on physics, not on geometry.

\section{Summary}

The 20th century essentially began with Einstein's relativistic $E=m c^{2}$ Lorentz transformation on time and space, differentiating "modern" physics from the pre-relativistic classical physics of Newtonian absolute space and time. The Lorentz rotation of time into space and space into time predicted "length contraction" and "time dilation", and led to the relativistic Hamiltonian of Equation (16). Muon physics and Hafele-Keating essentially nailed down physicist's belief in time dilation and length contraction despite that, per Rindler, "no direct experimental verification of length contraction has yet been attempted." Lorentz-based "velocity addition" has also been challenged [16] based on accelerator physics; the experimental basis of special relativity is more of a mixed bag than is perhaps generally recognized. Nevertheless no one seriously questions "time dilation" as an empirical fact. 
Yet while physics provides a means of understanding nature by focusing on changes in systems over time, our intuition provides a means of understanding focused on the direct perception of truth or fact, independent of any reasoning process. Mermin [17] says "... relativity reveals the nature of time to be shockingly different from what has been taken completely for granted." This mismatch between intuitive understanding of time and Einstein's relativistic theory of time is so pronounced that the journal Foundations of Physics last year published three papers [18] [19] [20] in a special issue focused on this problem.

Despite the lack of experimental verification of length contraction and of velocity addition, the Lorentz transformation is built-into modern physics at the root: the action principle and the Lagrangian formulation of physics are basic to all fields of physics and employ the Lorentz transformation from the beginning. This is almost unanimously interpreted as support for space-time rotation, although the excellent relativity text by Lucas and Hodgson summarizes the many derivations of the Lorentz transformation and concludes:

“... if we insist on retaining Newtonian dynamics, and the Newtonian definition of velocity and acceleration, then we can still obtain relativistically correct results if we pay the price of allowing the mass to depend on the velocity."

This is exactly the assumption behind our treatment of inertial clocks. However, the relativistic proposition of "no preferred frame" forbids this interpretation by systematically "resetting" inertial mass to rest mass in every frame, thereby "throwing away" the differential kinetic mass responsible for clock-slowing in our energy theory.

Although our assumption of absolute space was hinted at in Equation (1), there is a literature on "gravity as aether" that even Einstein appeared to subscribe to in later years. This assumption is strengthened by the 2017 detection of gravitational waves and light waves from inspiraling neutron stars, and also receives little-recognized support from Michelson-Gale experiments [21].

It was also noted in this paper that the Hafele-Keating "time dilation" experiments do not fit the special relativity theory as one must violate the no-preferredframe proposition in order to explain the East-West dichotomy.

In summary, we recall Hestenes remark on physical modeling:

"everything we know about physical space-time is known through its representation by some model, so when we are thinking about space-time and its properties, we' re actually thinking about the model. (...) however we attribute an independent existence to space-time which might not be accurately represented by our model (...) so we must keep the distinction clear when considering the possibility that the model is wrong."

When Hestenes says "we attribute an independent existence to ...", he is speaking of ontology. He is warning here that our "model" may not correspond to reality. Physicists who regard ontology as metaphysics are comfortable switching ontology as necessary, for example, invoking a forbidden preferred frame if needed to explain clock speedup when flying west. We note simply that our model, based on inertial clocks in absolute space and time, appears compati- 
ble with all experimental results. It is unlikely that nature maintains a geometric ontology and an inertial mass ontology, just for the satisfaction of physicists who have learned one model or another.

Our treatment of inertial clocks as simple harmonic oscillators, subject to thermodynamic analysis, has yielded dynamical behavior consistent with the experimental record. The approach is based on physical phenomena rather than geometry, and assumes universal time and preferred spatial frames associated with local center-of-mass. The result we have achieved is formulated in local absolute space, identified with local center-of-mass, and universal time, $t$. The theory is compatible with Hafele-Keating and with Pound-Rebka experiments and agrees with treatments of clock slowdown in special relativity and general relativity. In this mathematical theory of physics we have avoided some thorny ontological issues. For example, Poisson and Will's excellent gravity text states:

"Time flows at a rate that depends on position in a gravitational field."

Alternatively, one could state that time does not "flow" at that rate; instead, energy-time conservation dictates that inertial clocks measure time at that rate. The inertial mass of the clock oscillator incorporates the mass-equivalent of kinetic energy and the potential energy of the gravitational field; the resultant change in oscillator frequency is counted and the changed count represents the clock reading or time measurement. This thermodynamic interpretation establishes the common framework we sought for "clock slowing" in special and general relativity.

\section{Acknowledgements}

I am grateful to Otis Monty Frost, Stanley L. Robertson, A. Richard Zacher and Steven Kenneth Kauffmann for helpful conversations and criticisms.

\section{Conflicts of Interest}

The author declares no conflicts of interest regarding the publication of this paper.

\section{References}

[1] Rindler, W. (1991) Introduction to Special Relativity. 2nd Edition, Oxford University Press, New York.

[2] Ohanian, H. and Ruffini, R. (2013) Gravitation and Spacetime. 3rd Edition, Cambridge University Press, New York. https://doi.org/10.1017/CBO9781139003391

[3] Hafele, T. and Keating, R. (1972) Science, 177, 166-168. https://doi.org/10.1126/science.177.4044.166

[4] Pound, R. and Rebka, V. (1959) Physical Review Letters, 3, 439. https://doi.org/10.1103/PhysRevLett.3.439

[5] Abbott, B., et al. (2017) Physical Review Letters, 119, Article ID: 161101.

[6] Will, C. (2018) Theory and Experiment in Gravitational Physics. 2nd Edition, Cambridge Press, Cambridge.

[7] Hestenes, D. (1986) New Foundations for Classical Mechanics. 2nd Edition, Kluwer 
Academic Pub., Dordrecht. https://doi.org/10.1007/978-94-009-4802-0

[8] Susskind, L. (2012) Special Relativity Lecture 1. Stanford Video Series. https://youtu.be/toGH5BdgRZ4

[9] Lucas, J. and Hodgson, P. (1990) Space-Time and Electromagnetism. Clarendon Press, Oxford.

[10] Kauffmann, S. (2018) Dirac Theory's Breaches of Quantum Correspondence and Relativity; Nonrelativistic Pauli Theory's Unique Relativistic Extension.

https://vixra.org/abs/1810.0114 https://vixra.org/abs/1901.0228

[11] Reynolds, W. (1965) Thermodynamics. 2nd Edition, McGraw-Hill, New York.

[12] Hill, T. (1960) An Intro to Statistical Thermodynamics. Dover Publications, New York.

[13] Poisson, E. and Will, C. (2014) Gravity. Cambridge University Press, Cambridge.

[14] Robertson, S. (2019) Prespacetime Journal, 10, 621-626.

[15] Paige, A., Plato, A. and Kim, M. (2020) Physical Review Letters, 124, Article ID: 160602. https://doi.org/10.1103/PhysRevLett.124.160602

[16] Cannoni, M. (2016) Lorentz Invariant Relative Velocity.

[17] Mermin, D. (2005) It's about Time. Princeton University Press, Princeton. https://doi.org/10.1515/9781400830848

[18] Rovelli, C. (2019) Foundations of Physics, 49, 1325-1335. https://doi.org/10.1007/s10701-019-00312-9

[19] Thyssen, P. (2019) Foundations of Physics, 49, 1336-1354. https://doi.org/10.1007/s10701-019-00294-8

[20] Ben-Yami, H. (2019) Foundations of Physics, 49, 1355-1364. https://doi.org/10.1007/s10701-019-00306-7

[21] Klingman, E. (2018) Everything's Relative, or Is It? http://vixra.org/pdf/1812.0424v1.pdf 